\title{
Developmental Concerns of Parents and Unmet Healthcare Needs in Low-and Middle-Income Countries: Please Mind the Gap!
}

\author{
Bhavneet Bharti ${ }^{1} \cdot$ Prahbhjot Malhi $^{1}$
}

Received: 6 November 2020 / Accepted: 6 November 2020 / Published online: 16 November 2020

(C) Dr. K C Chaudhuri Foundation 2020

Recent 'Global Burden of Disease' data revealed that $8.4 \%$ of children younger than 5 y suffered developmental disabilities (95\% Uncertainty Interval: 7.7-9.1\%) contributing to nearly $13.5 \%$ of years lived with disability among all health conditions in children [1]. Moreover, parental concerns are known to be significant predictors of developmental risk among children $[2,3]$. In this backdrop, study by Yoldas TC et al. in the current issue makes an interesting and important reading as it draws attention to the unmet developmental needs among young children and the quality chasm for services provided to address these concerns in low- and middle-income countries [4]. The paper highlights that $28.8 \%$ of the caregivers had developmental concerns but only half of them had consulted a health professional leading to what the authors term as 'missed opportunities'. The authors have used 45-item questionnaire including translated items from PEDS (Parents Evaluation of Developmental Status) which is an evidence based developmental screening tool to elicit parental concerns in multiple developmental domains [4]. Various biological and psychosocial adverse factors at individual and population level are known to trigger parental concerns. Among parents who raised concerns in the current study, large proportions of children were accompanied by some disability/chronic illness (43.8\%). In addition, history of premature delivery (38.09\%) was also reported by parents of such children. So, there were already existing developmental risk factors which required medical attention. Indeed, health-care providers in busy settings are likely to miss or ignore the developmental concerns of families; therefore, simple screening tools need to be integrated in health-care systems. Another key finding of this study was significantly higher developmental concerns among

Bhavneet Bharti

bhavneetsahul@gmail.com

1 Department of Pediatrics, Advanced Pediatrics Centre, PGIMER, Chandigarh 160012, India parents who did not engage in active age appropriate play with their children. This basically underpins the importance of responsive and stimulating care which is crucial for promoting the cognitive, language, socio-emotional, and physical development of the child, particularly in impoverished homes [5, 6]. Play fosters stable and nurturing relationship between parent and child, which helps the child to ward off toxic stress. In this context, pediatricians are uniquely placed to educate and guide families about psychosocial stimulation of their children and thereby, increasing the opportunities for healthy parentchild interactions, exploration of the environment, and language stimulation to promote early child development. Growing body of literature documents that intervention programs are particularly efficacious in the early years of life and these positive benefits have long-term academic and economic pay-offs. Indeed, India had initiated Rasthriya Bal Swasthya Karyakaram (RBSK) that incorporates both screening and early intervention components both in the community as well as health-care settings in the year 2013. Under the umbrella of the RBSK, 'District Early Intervention Centre' (DEIC) have been set up, which specifically targets children under 6 y of age with the specific goal of increasing their developmental potential with comprehensive management, care and referral services. Widespread implementation of such interventions can play key role to promote, support, monitor and scale interventions promoting optimal early childhood development as envisaged in UN Sustainable Development Goals (SDGs).

\section{Compliance with Ethical Standards}

Conflict of Interest None.

\section{References}

1. Global Research on Developmental Disabilities Collaborators. Developmental disabilities among children younger than 5 years in 195 countries and territories, 1990-2016: a systematic analysis for 
the global burden of disease study 2016. Lancet Glob Health. 2018;6:e1100-21.

2. Woolfenden S, Eapen V, Williams K, Hayen A, Spencer N, Kemp L. A systematic review of the prevalence of parental concerns measured by the parents' evaluation of developmental status (PEDS)indicating developmental risk. BMC Pediatr. 2014;14:231-44.

3. Malhi P, Singhi P. Role of parents' evaluation of developmental status in detecting developmental delay in young children. Indian Pediatr. 2002;39:271-5.

4. Yoldaș TC, Özmert EN, Bayazıt Y, Tanrıkulu B, Yetim H, Çakır B. Developmental concerns, parental perceptions and missed opportunities from different levels of health centers in a middle-income country. Indian J Pediatr. 2020. https://doi.org/10.1007/s12098020-03302-2.

5. Jeong J, Pitchik HO, Yousafzai AK. Stimulation interventions and parenting in low- and middle-income countries: a meta-analysis. Pediatrics. 2018;141:e20173510.

6. Malhi P, Menon J, Bharti B, Sidhu M. Cognitive development of toddlers: does parent stimulation matter? Indian J Pediatr. 2018;85: 498-503.

Publisher's Note Springer Nature remains neutral with regard to jurisdictional claims in published maps and institutional affiliations. 